\title{
Comparison of toxic effects of some nonsteroidal anti- inflammatory medications on the kidney and lung tissues of rats
}

\author{
Manal N. Al-Hayder ${ }^{1}$, Tamadir H. W. Aledani ${ }^{2 *}$, Rafid A. Doulab ${ }^{3}$ \\ \{Manal.Abd-AlHassan@uobasrah.edu.iq ${ }^{1}$, tamadir.wadi@uobasrah.edu.iq ${ }^{2}$, \\ rafid.doulab@uobasrah.edu.iq $\left.{ }^{3}\right\}$
}

Department of Pharmacology \& Toxicology, College of Pharmacy, University of Basrah ${ }^{1}$, Department of Clinical Laboratory Sciences, College of Pharmacy, University of Basrah², Central laboratory, College of Pharmacy, University of Basrah ${ }^{3}$

\begin{abstract}
Nonsteroidal anti-inflammatory drugs that include diclofenac sodium and naproxen are frequently and widely used. Nevertheless, they cause adverse deleterious effects on multiple organs. This work represents a comparative study of the toxic effects of diclofenac sodium and naproxen in rats that is almost nonexistent.The methodology is applied on 15 rats orally administered nonsteroidal drugs for three weeks. The hematopathological and histopathological effects were evaluated. Abnormal hematological profile in both diclofenac sodium and naproxen treated groups comparing to the control is illustrated. Interestingly, the statistical comparison between the two treated groups revealed that in the naproxen group the red blood cells count, hemoglobin, and mean corpuscular hemoglobin concentration were significantly lower, while the platelets and plateletcrit significantly increased more than another group. The histopathological micrographs demonstrated the harmful effects of both drugs in the kidney and lung tissues. We concluded that a high dose of naproxen potentially generates higher toxic effects compared to a lower dose of diclofenac sodium as habitually used.
\end{abstract}

Keywords: Blood parameters, histopathology, nonsteroidal anti-inflammatory drugs, toxic effects.

\section{Introduction}

Nonsteroidal anti-inflammatory drugs (NSAID) are known as cyclooxygenase inhibitors and are widely used as analgesics, anti-pyretics, anti-inflammatory and anti-cancer drugs to treat different types of cancer, chronic pain, postoperative pain, rheumatoid arthritis, osteoarthritis, and spasms [1, 2, 3]. As well as, there are many recent speculations to use NSAID in the therapy of COVID-19 such as indomethacin for blocking coronavirus RNA synthesis. It is also shown that it is possible to use celecoxib and diclofenac for inhibiting cyclooxygenase- 2 which is promoted by SARS coronavirus [4]. Although this considerable therapeutic use of the NSAID, they cause adverse harmful effects on multiple organs such as gastrointestinal, cerebral, cardiovascular, renal, hepatic, and pulmonary pathologies that can lead to the risk of organ damage especially when they are daily used in high dose and for long term $[1,2]$. Thus, diclofenac sodium and naproxen among the NSAID are the most available, cheapest, and most extensively and frequently used. Several studies have shown their toxicities in the rats including gastrointestinal ulceration, gastric damage, severe lesions in the liver and cerebrum, hepato-renal damage, and acute pulmonary toxicity $[5,6,7,8,9,10]$. 
Regarding the systematic reviews and meta-analysis to compare between the effects of diclofenac sodium and naproxen in the human $[11,12,13]$, the comparative study of their toxic effects in the rats is almost nonexistent according to our knowledge. Therefore, the aim of the present study is to shed more light on the hematopathological and histopathological effects of diclofenac sodium and naproxen in the kidney and lung tissues of the rats as a comparative study.

\section{Materials and methods}

\subsection{Experimental animals and design}

In this study, fifteen males of the albino rats, 5 weeks old and weighing 70-80g, were used. These animals were housed under typical conditions of the temperature and lighting in the animals' house of Pharmacy College, University of Basrah, Iraq. Then they were distributed to three groups: control, treated with diclofenac sodium and treated with naproxen in each group of five rats.

All animals were weighed weekly and orally administered by gavage every day for three weeks. The second group was administered $5 \mathrm{mg} / \mathrm{kg}$ body weight (b.w.)/day of the $50 \mathrm{mg}$ diclofenac sodium tablet. Whereas, the third group was administered $50 \mathrm{mg} / \mathrm{kg}$ b.w./day of the $500 \mathrm{mg}$ naproxen tablet. Both diclofenac sodium and naproxen were dissolved in 5\% dimethyl sulfoxide (DMSO). Thus, the control group was administered 0.1ml DMSO/day.

\section{2 Hematological estimation}

At the termination of the third week, using chloroform the rats were anesthetized to collect blood samples by cardiac puncture in collection tubes containing ethylenediaminetetraacetic acid (EDTA) as an anticoagulant. The automated blood cell analyzer (Genex, Florida, USA) was used for evaluation directly all blood parameters (total white blood cell count, granulocytes, mean corpuscular volume, monocytes count, red cell distribution width, platelet large cell ratio, plateletcrit, platelets, lymphocytes, red blood cells count, hematocrit, mean corpuscular hemoglobin concentration, hemoglobin, and mean corpuscular hemoglobin).

\section{3 Histopathological examination}

After euthanasia and dissection, kidneys and lungs were preserved in $10 \%$ formaldehyde until embedding in the paraffin wax. The rotary microtome was used to cut the tissue samples into $5 \mu \mathrm{m}$ sections. Subsequently, for microscopic examination, a haematoxylin and eosin (H\&E) stain was utilized.

\subsection{Hematological data analysis}

Statistical GraphPad Prism software using one-way analysis of variance then Bonferroni's multiple comparison test analyzed the hematological results. Also, an unpaired t-test was used for comparison between diclofenac sodium and naproxen. The significance was assessed at $\mathrm{p}<0.05$. 


\section{Results}

\subsection{Hematological parameters}

As shown in Figure (1), the hematological profile of the treated rats in comparison with the control showed abnormalities in the whole blood picture for both diclofenac sodium and naproxen. The values of total white blood cell count, granulocytes, mean corpuscular volume, monocytes count (MID), red cell distribution width, platelet large cell ratio (P-LCR), plateletcrit (PCT), and platelets (PLT) were elevated. This elevation was significant for MID, PLT and PCT in the rats treated with diclofenac sodium and those treated with naproxen. Whereas P-LCR was significantly affected only in the rats treated with naproxen.

In contrast, the values of lymphocytes, red blood cells count (RBC), hematocrit (HCT), mean corpuscular hemoglobin concentration (MCHC), hemoglobin (HGB) and mean corpuscular hemoglobin $(\mathrm{MCH})$ were decreased compared to the control. Significantly, RBC was diminished in the rats treated with diclofenac sodium and in those treated with naproxen. For lymphocytes, its significant decreased value was remarked only in the diclofenac sodium-treated rats. While HGB, $\mathrm{HCT}, \mathrm{MCH}$, and MCHC were significantly impacted only in the rats treated with naproxen (Figure $1)$.

Remarkably, the statistical analysis showed that the values of RBC, HGB, and MCHC were significantly lesser in the rats administered naproxen than with diclofenac sodium. Also, PLT and PCT were significantly augmented in the naproxen-treated rats than those with diclofenac sodium (Figure 2). 


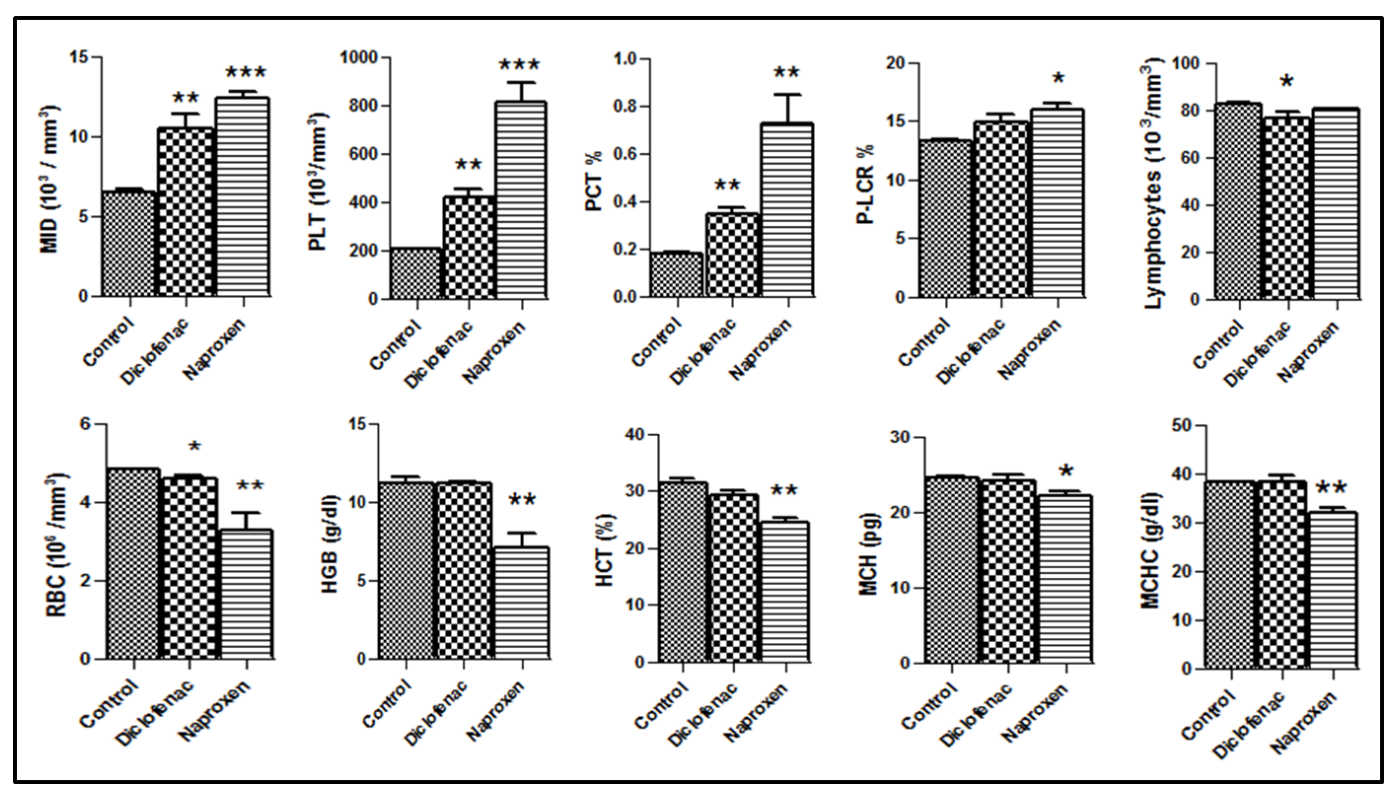

Figure 1. Significant impact of diclofenac sodium and naproxen on blood parameters compared to the control in the albino rats. MID: monocytes count, PLT: platelets, PCT: plateletcrit, P-LCR: platelet-large cell ratio, RBC: red blood cells count, HGB: hemoglobin, HCT: hematocrit, $\mathrm{MCH}$ : mean corpuscular hemoglobin, and MCHC: mean corpuscular hemoglobin concentration. For expressing the significant values; * for $\mathrm{p}<0.05$, ** for $\mathrm{p}<0.01$, and $* * *$ for $\mathrm{p}<0.001$ as mean \pm SEM values.

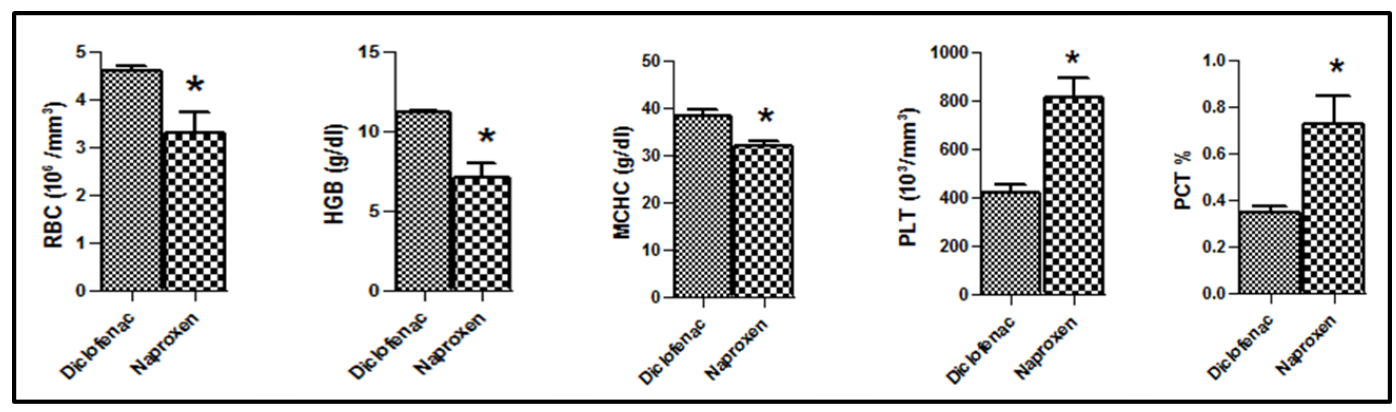

Figure 2. Significant impact of naproxen on some hematological parameters in comparison with diclofenac sodium in the albino rats. RBC: Red blood cells count, HGB: hemoglobin, MCHC: mean corpuscular hemoglobin concentration, PLT: platelets, and PCT: plateletcrit. Mean \pm SEM expresses the values; the significance is expressed as $* \mathrm{p}<0.05$. 


\subsection{Histopathological findings}

Histological examination of the kidney tissue in the control group exhibited normal glomeruli, proximal and distal tubules. In contrast, the kidneys' sections of both groups treated with diclofenac sodium and with naproxen illustrated harmful changes including necrosis in the epithelial tissue of tubules, shrinkage of the glomeruli, and enlargement of the Bowman's space (Figure 3).

Moreover, the photomicrographs of the lung tissue also revealed evident pathological changes in both treated groups involving alveoli surrounded by thickening walls, and intra- alveolar hemorrhage comparing to the control which showed normal architecture of lung tissue as normal bronchioles and alveoli structure with thin inter-alveolar walls (Figure 4).

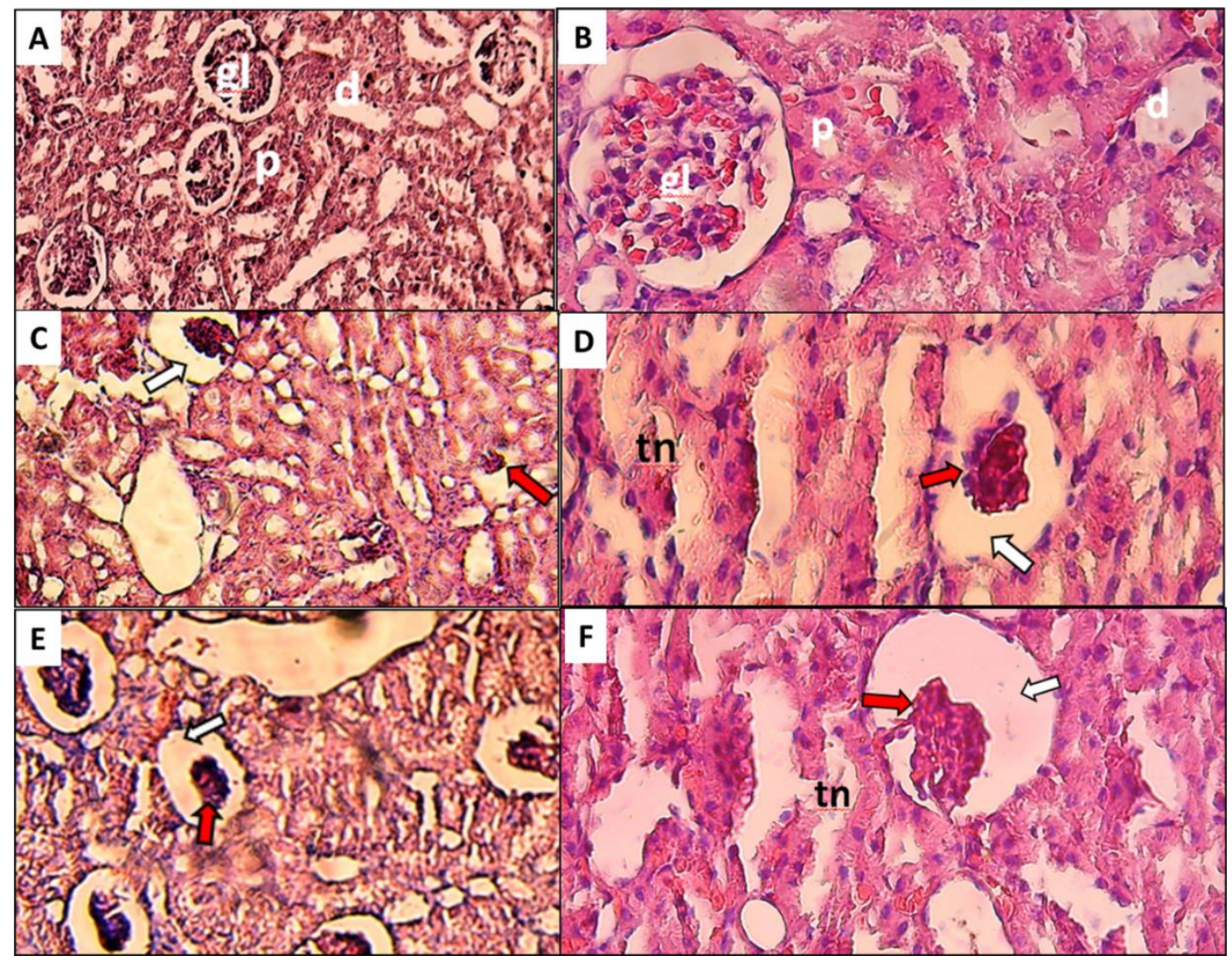

Figure 3. Photomicrographs of the kidney tissue in albino rats. (A\&B) Control group showed normal kidney histology: normal glomerulus (gl), proximal (p) and distal tubules (d). (C\&D) Diclofenac sodium-treated group revealed glomerular shrinkage (red arrow), Bowman's space enlargement (white arrow), and tubular necrosis (tn). (E\&F) Naproxen-treated group also exhibited glomerular shrinkage (red arrow), Bowman's space 


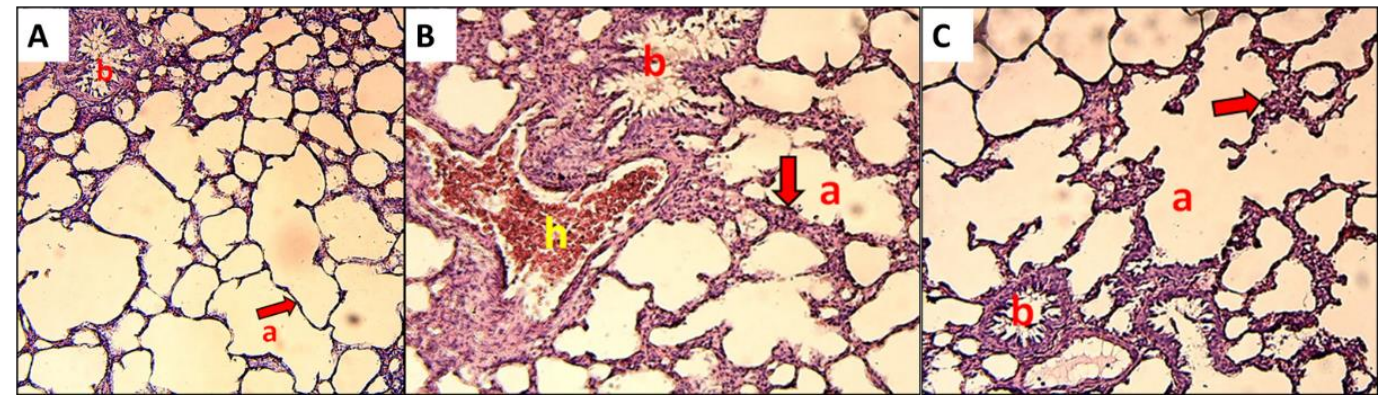

Figure 4. Photomicrographs of the lung tissue in albino rats. (A) Control group displayed normal lung histology: normal structure of the alveoli (a) enclosed by thin septa (red arrow), and normal bronchioles (b). (B) and (C) Diclofenac sodium-treated group and naproxen-treated group respectively elucidated thickened alveolar septa (red arrow), and intra- alveolar hemorrhage (h). Using H\&E stain and magnification 200x.

\section{Discussion}

The increasing demand for the NSAID to treat a broad spectrum of diseases encourages investigating the least harm and toxicity for different organs. In this regard, NSAID can cause increasing and dysregulation of inflammatory response that induce pathologic harms [14]. Thus, the findings for both diclofenac sodium and naproxen demonstrated hematological alterations that were represented by significant elevation of MID, PLT, and PCT in comparison to the control. The significant increase in the platelets may indicate their aggregation to prevent potential bleeding in the site of the damage. Also, there was a significant decrease in lymphocytes, RBC, HCT, MCH, $\mathrm{HGB}$, and MCHC. According to previous studies [15, 16, 17], the significant decrease in the RBC, $\mathrm{HGB}, \mathrm{HCT}, \mathrm{MCH}, \mathrm{MCHC}$, and lymphocytes considers as an indicator of hemolytic anemia and it is correlated with toxicity.

On the other hand, the histopathological findings of this study confirmed the toxic effects of both diclofenac sodium and naproxen. The hemorrhage in the tissues possibly interprets the significant decrease in the RBC and hemoglobin parameters; also it could explain the significant increase in the platelets. For diclofenac sodium, the results of lung histopathology are similar to those obtained by Aljuhani et al. [10], but they intraperitoneally administered the albino rats with overdose $(200 \mathrm{mg} / \mathrm{kg}$ b.w.) of diclofenac. Also, the diclofenac-induced nephrotoxic injury in the kidney tissue was achieved in the albino rats by intraperitoneal administration with $50 \mathrm{mg} / \mathrm{kg}$ b.w. $[9,18]$. In this context, there are no obtainable data around the histopathological effects of naproxen in kidney and lung tissues of the rats for supporting the interpretation of our results. Despite that, naproxeninduced renal toxicity and lung lesions in humans are well known [19, 20, 21, 22]. There is a case report of 17 years old girl taken 4 times/day of $250 \mathrm{mg}$ naproxen for 4 days; the biopsy from her 
kidney appeared interstitial nephritis and necrosis in the papillae and tubules [23].

\section{Conclusion}

Diclofenac sodium and naproxen had shown deleterious hematopathological and histopathological effects in the rats. The hematopathological changes could cause and reflect tissue damage involving the kidney and the lung. Furthermore, both drugs had certain convergent effects. However, the outcomes suggest potential toxic effects of naproxen more than diclofenac sodium as a consequence of using it in a higher dose than diclofenac; that was evident from the statistical significance.

Acknowledgments. All authors thank the deanship of the College of Pharmacy, University of Basrah.

\section{References}

[1] Dong Y-H, Chang C-H, Wu L-C, et al. Comparative cardiovascular safety of nonsteroidal antiinflammatory drugs in patients with hypertension: a population-based cohort study. Br J Clin Pharmacol 2018; 84: 1045-1056.

[2] Bindu S, Mazumder S, Bandyopadhyay U. Non-steroidal anti-inflammatory drugs (NSAIDs) and organ damage: A current perspective. Biochem Pharmacol 2020; 180: 114147.

[3] Liggett JL, Zhang X, Eling TE, et al. Anti-tumor activity of non-steroidal anti-inflammatory drugs: cyclooxygenase-independent targets. Cancer Lett 2014; 346: 217-224.

[4] FitzGerald GA. Misguided drug advice for COVID-19. Science 2020; 367: 1434.

[5] Kim J-H, Kim Y-S, Song G-G, et al. Protective effect of astaxanthin on naproxen-induced gastric antral ulceration in rats. Eur J Pharmacol 2005; 514: 53-59.

[6] Silva RO, Santana APM, Carvalho NS, et al. A sulfated-polysaccharide fraction from seaweed Gracilaria birdiae prevents naproxen-induced gastrointestinal damage in rats. Mar Drugs 2012; 10: $2618-2633$.

[7] Ekundi-Valentim E, Mesquita FP, Santos KT, et al. A comparative study on the anti-inflammatory effects of single oral doses of naproxen and its hydrogen sulfide (H2S)-releasing derivative ATB-346 in rats with carrageenan-induced synovitis. Med Gas Res 2013; 3: 24.

[8] Lojo N, Rasic Z, Zenko Sever A, et al. Effects of Diclofenac, L-NAME, L-Arginine, and Pentadecapeptide BPC 157 on Gastrointestinal, Liver, and Brain Lesions, Failed Anastomosis, and Intestinal Adaptation Deterioration in 24 Hour-Short-Bowel Rats. PLoS One 2016; 11: e0162590. 
[9] Mostafa RE, El-Marasy SA, Abdel Jaleel GA, et al. Protective effect of royal jelly against diclofenac-induced hepato-renal damage and gastrointestinal ulcerations in rats. Heliyon 2020; 6: e03330.

[10] Aljuhani N, Elkablawy MA, Elbadawy HM, et al. Protective effects of Ajwa date extract against tissue damage induced by acute diclofenac toxicity. J Taibah Univ Med Sci 2019; 14: 553-559.

[11] McGettigan P, Henry D. Cardiovascular risk with non-steroidal anti-inflammatory drugs: systematic review of population-based controlled observational studies. PLoS Med 2011; 8: e1001098.

[12] van Walsem A, Pandhi S, Nixon RM, et al. Relative benefit-risk comparing diclofenac to other traditional non-steroidal anti-inflammatory drugs and cyclooxygenase- 2 inhibitors in patients with osteoarthritis or rheumatoid arthritis: a network meta-analysis. Arthritis Res Ther 2015; 17: 66.

[13] Coxib and traditional NSAID Trialists' (CNT) Collaboration, Bhala N, Emberson J, et al. Vascular and upper gastrointestinal effects of non-steroidal anti-inflammatory drugs: meta-analyses of individual participant data from randomised trials. Lancet 2013; 382: 769-779.

[14] Maseda D, Zackular JP, Trindade B, et al. Nonsteroidal Anti-inflammatory Drugs Alter the Microbiota and Exacerbate Clostridium difficile Colitis while Dysregulating the Inflammatory Response. mBio; 10. Epub ahead of print 8 January 2019. DOI: 10.1128/mBio.02282-18.

[15] Orinya OA, Adenkola AY, Ogbe RJ. Haematological and biochemical studies on the effect of diclofenac sodium on Wistar Rattus norvegicus. Int J Bio Chem Sci 2017; 10: 2231.

[16] Agha FE, El-Badry MO, Hassan DAA, et al. Role of Vitamin E in Combination with Methionine and L- carnosine Against Sodium Fluoride-Induced Hematological, Biochemical, DNA Damage, Histological and Immunohistochemical Changes in Pancreas of Albino Rats. Life Science Journal 2012; 9: 1260-1275.

[17] Khan AM, Dubey N, Raina R, et al. TOXIC EFFECTS OF DELTAMETHRIN AND FLUORIDE ON HEMATOLOGICAL PARAMETERS IN RATS. Research Report 46(1), Jammu, India: Department of Pharmacology and Toxicology, Faculty of Veterinary and Animal Sciences, Shere-Kashmir University of Agricultural Sciences and Technology.

[18] Peter S J, Basha S K, Giridharan R, et al. Suppressive effect of Spirulina fusiformis on diclofenacinduced hepato-renal injury and gastrointestinal ulcer in Wistar albino rats: A biochemical and histological approach. Biomed Pharmacother 2017; 88: 11-18.

[19] Hörl WH. Nonsteroidal Anti-Inflammatory Drugs and the Kidney. Pharmaceuticals (Basel) 2010; 3 : 2291-2321.

[20] Whelton A, Schulman G, Wallemark C, et al. Effects of celecoxib and naproxen on renal function in the elderly. Arch Intern Med 2000; 160: 1465-1470.

[21] Ogawa H, Kurashima K, Namura M, et al. Pulmonary infiltrates with eosinophilia due to naproxen. Jpn J Med 1991; 30: 32-34. 
[22] Guerrero Gómez DA, París Zorro S, Aponte Barrios W, et al. Drug reaction with eosinophilia and systemic symptoms (DRESS) with severe and atypical lung involvement. Radiol Case Rep 2020; 15 : $2178-2182$.

[23] Kovacevic L, Bernstein J, Valentini RP, et al. Renal papillary necrosis induced by naproxen. Pediatr Nephrol 2003; 18: 826-829. 